\title{
Joint Trajectory and Power Allocation Design for Secure Artificial Noise aided UAV Communications
}

This paper was downloaded from TechRxiv (https://www.techrxiv.org).

\section{LICENSE}

CC BY 4.0

\section{SUBMISSION DATE / POSTED DATE}

02-01-2021 / 07-01-2021

\section{CITATION}

Tatar Mamaghani, Milad; Hong, Yi (2021): Joint Trajectory and Power Allocation Design for Secure Artificial Noise aided UAV Communications. TechRxiv. Preprint. https://doi.org/10.36227/techrxiv.13264745.v1

$\mathrm{DOI}$ 


\title{
Joint Trajectory and Power Allocation Design for Secure Artificial Noise aided UAV Communications
}

\author{
Milad Tatar Mamaghani ${ }^{\circledR}$, Graduate Student Member, IEEE, Yi Hong ${ }^{\circledR}$, Senior Member, IEEE \\ Electrical and Computer Systems Engineering Department, Monash University, Melbourne, Australia
}

\begin{abstract}
This paper investigates an average secrecy rate (ASR) maximization problem for an unmanned aerial vehicle (UAV) enabled wireless communication system, wherein a UAV is employed to deliver confidential information to a ground destination in the presence of a terrestrial passive eavesdropper. By employing an artificial noise (AN) injection based secure two-phase transmission protocol, we aim at jointly optimizing the UAV's trajectory, network transmission power, and AN power allocation over a given time horizon to enhance the ASR performance. Specifically, we divide the original non-convex problem into four subproblems, and propose a successive convex approximation based efficient iterative algorithm to solve it suboptimally with guaranteed convergence. Simulation results demonstrate significant security advantages of our designed scheme over other known benchmarks, particularly for stringent flight durations.
\end{abstract}

Index Terms-UAV communications, physical layer security, artificial noise injection, convex optimization.

\section{INTRODUCTION}

$\mathrm{R}$ ECENTLY, unmanned aerial vehicle (UAV) aided wireless communications have attracted a great deal of research interests. UAV has unique attributes such as flexible deployment, dominant line-of-sight (LoS) air-ground (AG) channel, and controlled mobility in three-dimensional (3D) space. As a result, UAV can act as either a flying base station (BS), aerial radio access point, or aerial relay via $A G$ links to expand coverage, provide seamless connectivity, and support highrate communications. However, the open nature of AG links inevitably makes such systems vulnerable to eavesdropping attacks, and hence, safeguarding UAV communication is of significant importance [1].

Physical layer security (PLS) techniques have been intensively studied in the past decade for terrestrial wireless communications with fixed or quasi-stationary nodes against eavesdropping (see [2], [3], and references therein). In the recent few years, a large number of research studies have been viewed UAV communications from the PLS perspective (see [4] and references therein). Particularly, Zhang et al. in [5] have considered a three-node scenario and investigated UAV's trajectory design and transmission power control for both downlink and uplink UAV communications in the presence of a passive eavesdropper. Robust trajectory and power design of UAV communications against multiple ground eavesdroppers with imperfect locations has been explored in [6]. In [7], the authors have investigated a secrecy rate maximization problem of the UAV-mobile relaying in the presence of an adversary. This work has been later extended by considering the UAV's trajectory design in [8] and the energy efficiency design in
[9]. The authors in [10] proposed an energy-efficient lowaltitude secure UAV relaying scheme with destination-assisted jamming and energy harvesting. Leveraging the technique of employing an additional UAV as a mobile jammer has been studied in [11] to improve the secrecy of ground wiretap channel, and in [12]-[14], to enhance the security for the UAVBS scenario.

On the other hand, artificial noise (AN) based PLS, originally proposed in [15], is a popular technique that can efficiently combat eavesdropping for terrestrial communication systems (e.g., [16] for multiple-antenna systems, and [17] for a single-antenna system). In [17], a two-phase transmission scheme was proposed in the presence of a passive eavesdropper: in phase I, the legitimate receiver broadcasts $A N$, and in phase II, the transmitter forwards the received signal from Phase I along with the information signal to the legitimate receiver. Then the receiver removes the $\mathrm{AN}$ and recovers the information, while the wiretap link can be degraded due to AN injection. To the best of our knowledge, such a technique has never been examined for UAV communications considering associated new degrees of freedom, e.g., AG links and mobility.

In this paper, motivated by the abovementioned works, we propose a secure UAV communication scheme by adapting a single-antenna AN-injection technique to UAV-enabled wireless communications. Different from [17] that assumes static and fixed network nodes, our paper exploits the UAV's highmaneuverability and investigates secure UAV communications to maximize achievable average secrecy rate (ASR). Our work is also different from [5], since we employ a secure communication protocol via $\mathrm{AN}$-assisted resource allocation rather than simple direct transmission. Overall, the central innovation of this work lies in the joint design of UAV's trajectory and system power allocation for the proposed scheme, which is also capable of achieving perfect secrecy [2]. Our contributions are summarized below.

- Since the considered ASR maximization problem is intractable, we split this problem into subproblems according to the block coordinate descent (BCD) algorithm, solving each separately, as a compromise approach.

- We then propose a fast converging and computationally efficient iterative algorithm based on the successive convex approximation (SCA) method, to find a local optima with guaranteed convergence.

- For the proposed algorithm, we conduct convergence and complexity analysis, and show that the solution can be obtained in polynomial time order, making it applicable 
to UAV-based scenarios.

- By simulations, we demonstrate that our proposed algorithm can significantly improve ASR and achieve a notably higher secrecy performance compared to other benchmarks.

\section{SYSTEM MODEL AND PROBLEM FORMULATION}

We consider a UAV-based wireless communication system including Alice, Bob, and Eve (see Fig. 1), all equipped with single antenna operating in half-duplex mode. We assume that Bob and Eve have fixed locations with three-dimensional (3D) Cartesian coordinates $\omega_{b}=\left(x_{b}, y_{b}, 0\right)$ and $\omega_{e}=\left(x_{e}, y_{e}, 0\right)$, respectively, which are known to Alice. We define Alice's flying duration as $T$ seconds and her constant flying height as $H$ meters. Note that UAV's altitude can be time-varying in general, nonetheless, from a practical perspective, this parameter here is considered fixed and chosen to be the minimum allowable height for the purpose of I) avoiding energy consumption of UAV when rising/falling during the mission II) keeping the UAV away from any possible collision with environmental buildings and surroundings. This leads to establishing likely LoS links with ground terminals. Furthermore, Alice's $3 D$ location at time $t \in[0, T]$ is given by $\omega_{a}(t)=\left(x_{a}(t), y_{a}(t), H\right) \in \mathbb{R}^{1 \times 3}$. We adopt the widely used LoS-dominated AG links assumption (see previous research works and references [5], [14], [18]), since it offers a good approximation to AG links, particularly for rural areas [19]. Assuming that the Doppler effect arising from UAV's mobility is perfectly compensated at the terrestrial node $g \in\{b, e\}$, the channel power gain between Alice and any ground terminal is $\tilde{h}_{a g}(t)=\frac{\beta_{0}}{\left\|\omega_{a}(t)-\omega_{g}\right\|^{2}}$, where $\beta_{0}=\left(\frac{C}{4 \pi f_{c}}\right)^{2}$ denotes the reference channel power gain for unit distance, wherein $C$ is the speed of light, $f_{c}$ is the carrier frequency. Further, we have $\omega_{g}=\left(x_{g}, y_{g}, 0\right) \in \mathbb{R}^{1 \times 3}$, and $\|\cdot\|$ denotes the $L_{2}$-norm operator.

Descretizing Alice's flying time horizon into $N$ equalduration time slots, i.e., $\delta_{t} \triangleq \frac{T}{N}$, with $\delta_{t}$ representing slot length, Alice's $3 D$ continuous trajectory over $T$, i.e., $\omega(t)$ can be approximated by the sequence $\left\{\omega_{a}[n]\right\}_{n=1}^{N}$, such that $\omega_{a}[n]=\left(x_{a}[n], y_{a}[n], H\right), \quad \forall n \in \mathbb{N}$, where $\mathbb{N}=$ $\{1,2, \cdots, N\}$. Note that by choosing $\delta_{t}$ to be sufficiently small, we can consider that Alice's 3D location is approximately unchanged within each time slot, satisfying an invariant channel condition. Besides, we define her predetermined initial and final locations as $\omega_{a i} \in \mathbb{R}^{1 \times 3}$ and $\omega_{a f} \in \mathbb{R}^{1 \times 3}$, respectively. Letting $\bar{d}_{\delta} \triangleq \bar{V} \delta_{t}$, wherein $\bar{V}$ represents Alice's maximum speed, and accordingly, $\bar{d}_{\delta}$ denotes Alice's maximum displacement per time slot, yields the mobility constraints as

$$
\begin{array}{ll}
\text { C1 : } & \left\|\omega_{a}[1]-\omega_{a i}\right\| \leq \bar{d}_{\delta}, \\
\text { C2 : } & \left\|\omega_{a}[n+1]-\omega_{a}[n]\right\| \leq \bar{d}_{\delta}, \quad \text { for } n \in \mathbb{N} \backslash N \\
\text { C3 : } & \left\|\omega_{a f}-\omega_{a}[N]\right\| \leq \bar{d}_{\delta}, \\
& \text { III TRANSMISSION PRotocol }
\end{array}
$$

We present a UAV-assisted AN injection based two-phase transmission protocol. We apply frequency division duplexing (FDD) such that both phases of transmission are conducted simultaneously during the coherence time of the wireless channel with equally shared bandwidth.

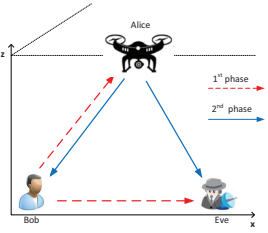

Fig. 1: AN-aided secure UAV communications system model.

In the first phase, at time slot $n$, Bob broadcasts an unknown noise-like signal $z[n]$ (known as pseudo random AN [17]) of unit-power, i.e., $\mathbb{E}\left\{\|z[n]\|^{2}\right\}=1$, where $\mathbb{E}\{\cdot\}$ is the expectation operator. This signal $z[n]$ is weighted with $\sqrt{P_{b}[n]}$ to result in transmitted power $P_{b}[n]$ per transmission. Thus, Alice and Eve respectively receive

$$
\begin{aligned}
y_{a}^{(1)}[n] & =\sqrt{P_{b}[n] \tilde{h}_{b a}[n]} z[n]+\nu_{a}[n], & \forall n \in \mathbb{N} \\
y_{e}^{(1)}[n] & =\sqrt{P_{b}[n]} g_{b e}[n] z[n]+\nu_{e}[n], & \forall n \in \mathbb{N}
\end{aligned}
$$

where the ground-to-ground channel power gain follow exponential distribution [3], i.e., $\left\|g_{b e}[n]\right\|^{2} \sim \operatorname{Exp}\left(\lambda_{b e}\right)$ with the scale parameter $\lambda_{b e}=\frac{\beta_{0}}{\left\|\omega_{b}-\omega_{g}\right\|^{\eta}}$ wherein $\eta>2, \nu_{a}, \nu_{e} \sim$ $\mathcal{N}\left(0, N_{0}\right)$ are the additive white Gaussian noise (AWGN) with the noise power $N_{0}$. Assuming that Bob sends no pilots (training sequences) alongside with $z[n]$, Alice and Eve both are unable to obtain $z[n]$.

In the second phase, at the same time slot, Alice generates

$$
x_{a}[n]=\sqrt{\alpha[n]} s[n]+\sqrt{1-\alpha[n]} \frac{y_{a}^{(1)}[n]}{\sqrt{\mathbb{E}\left\{\left\|y_{a}^{(1)}[n]\right\|^{2}\right\}}},
$$

where $s[n]$ is the unit-power information signal and $\alpha[n]$ is the power allocation factor satisfying

$$
\mathrm{C} 4: \quad 0 \leq \alpha[n] \leq 1, \quad \forall n \in \mathbb{N}
$$

Thereafter, Alice forwards information-bearing signal $x_{a}[n]$ with transmit power $P_{a}[n]$ over the channel $\tilde{h}_{a g}[n]$, yielding

$$
\begin{aligned}
& \tilde{y}_{g}^{(2)}[n]=\underbrace{\sqrt{\alpha[n] P_{a}[n] \tilde{h}_{a g}[n]} s[n]}_{\text {Information-bearing signal }}+\underbrace{\sqrt{\frac{(1-\alpha[n]) P_{a}[n] P_{b}[n] \tilde{h}_{b a}[n] \tilde{h}_{a g}[n]}{P_{b}[n] \tilde{h}_{b a}[n]+N_{0}}} z[n]}_{\text {AN interference }} \\
& +\underbrace{\sqrt{\frac{(1-\alpha[n]) P_{a}[n] \tilde{h}_{a g}[n]}{P_{b}[n] \tilde{h}_{b a}[n]+N_{0}}} \nu_{a}[n]+\nu_{g}[n]}_{\text {Noise }}, \quad \forall n \in \mathbb{N}
\end{aligned}
$$

where $\nu_{g} \sim \mathcal{N}\left(0, N_{0}\right)$ is the AWGN.

In practice, Alice and Bob's transmission powers are subject to the peak powers $\hat{P}_{a}$ and $\hat{P}_{b}$ and the average powers $\bar{P}_{a}$ and $\bar{P}_{b}$ constraints for all time slots $\forall n \in \mathbb{N}$ respectively as

$$
\begin{aligned}
& \text { C5 : } \frac{1}{N} \sum_{n=1}^{N} P_{a}[n] \leq \bar{P}_{a}, \quad \text { C6 }: 0 \leq P_{a}[n] \leq \hat{P}_{a}, \\
& \text { C7 : } \frac{1}{N} \sum_{n=1}^{N} P_{b}[n] \leq \bar{P}_{b}, \quad \text { C8 }: 0 \leq P_{b}[n] \leq \hat{P}_{b},
\end{aligned}
$$

To make the constraints $\mathrm{C} 5$ and $\mathrm{C} 7$ non-trivial, we assume $\bar{P}_{a}<\hat{P}_{a}$ and $\bar{P}_{b}<\hat{P}_{b}$. Letting $\gamma_{0} \triangleq \frac{\beta_{0}}{N_{0}}$, for convenience, we define $h_{a g}[n] \triangleq \frac{\tilde{h}_{a g}[n]}{N_{0}}$, which can be rewritten as

$$
h_{a g}[n]=\frac{\gamma_{0}}{\left(x_{a}[n]-x_{g}\right)^{2}+\left(y_{a}[n]-y_{g}\right)^{2}+H^{2}} .
$$


In contrast to Bob, Alice sends some pilot symbols prior to the data transmission in each time slot to enable receivers to perform channel estimation, and assuming that Bob can perfectly estimate $\hat{h}_{a b}$, the perfect channel state information (CSI) is available at him due to channel reciprocity assumption, i.e., $\hat{h}_{a b}=\hat{h}_{b a}$, similar to [3], [5], [8]. Further, owing to that Bob's generated $z[n], P_{b}[n]$ are known by himself, and we assume that the other parameters $\left(\alpha[n], P_{a}[n], N_{0}\right)$, all of which having fixed values in a given time slot, are publicly shared with Bob by Alice prior to the data transmission [17], Bob is able to remove AN, yielding his signal-to-interference-plus-noise ratio (SINR) as

$$
\gamma_{B}[n]=\frac{\alpha[n] P_{a}[n] h_{a b}[n]\left(P_{b}[n] h_{a b}[n]+1\right)}{\left[P_{b}[n]+(1-\alpha[n]) P_{a}[n]\right] h_{a b}[n]+1} .
$$

However, Eve has no information about the weighted $z[n]$, nor is she able to successfully decode $z[n]$ via, for example, joint processing of received signals in two phases, following the simple receiver architecture assumption in this work. Thus, the AN term cannot be cancelled out from (6), leading to

$$
\gamma_{E}[n]=\frac{\alpha[n] P_{a}[n] h_{a e}[n]}{(1-\alpha[n]) P_{a}[n] h_{a e}[n]+1} .
$$

According to [10], the instantaneous secrecy rate of the proposed UAV-aided communications in $\mathrm{bps} / \mathrm{Hz}$ is defined as

$$
R_{\text {sec }}[n] \triangleq \frac{1}{2}\left[C_{B}[n]-C_{E}[n]\right]^{+}, \quad \forall n \in \mathbb{N}
$$

with $C_{B}[n] \triangleq \log _{2}\left(1+\gamma_{B}[n]\right)$ and $C_{E}[n] \triangleq \log _{2}\left(1+\gamma_{E}[n]\right)$, where $[x]^{+} \triangleq \max \{x, 0\}$, and fraction $\frac{1}{2}$ is due to halfduplexing. Defining $\boldsymbol{\omega}_{\boldsymbol{a}} \triangleq\left\{\omega_{a}[n]\right\}_{n=1}^{N}, \quad \boldsymbol{\alpha} \triangleq\{\alpha[n]\}_{n=1}^{N}$, $\mathbf{P}_{\mathbf{a}} \triangleq\left\{P_{a}[n]\right\}_{n=1}^{N}$, and $\mathbf{P}_{\mathbf{b}} \triangleq\left\{P_{b}[n]\right\}_{n=1}^{N}$ we focus on maximizing the average secrecy rate (ASR) of the proposed scheme over $N$ time slots formulated as $N$

$$
\begin{gathered}
(\mathrm{P}): \max _{\mathbf{P}_{\mathbf{a}}, \mathbf{P}_{\mathbf{b}}, \boldsymbol{\alpha}, \boldsymbol{\omega}_{\boldsymbol{a}}} \\
\text { s.t. } \quad \mathrm{C} 1-\mathrm{C} 8,
\end{gathered}
$$

The optimization problem $(\mathrm{P})$ is challenging to solve due to non-concave as well as non-differentiable objective function owing to the operator $[\cdot]^{+}$. At the optimal point, $[\cdot]^{+}$can be removed from $R_{s e c}[n]$ (otherwise, by setting $\mathbf{P}_{\mathbf{a}}=\mathbf{0}$ one can obtain zero ASR), making at least differentiable objective function. Nonetheless, it is still unsolvable due to being a nonconvex problem with respect to the optimization variables.

\section{PROPOSED ITERATIVE ALGORITHM}

To solve problem $(\mathrm{P})$, we propose a computationally tractable iterative algorithm based on BCD and SCA such that problem $(\mathrm{P})$ is split into four subproblems wherein at each time, we optimize one block of variables using convex optimization while keeping the others unchanged in an alternative manner until convergence. To detail the procedure, we omit the constant factor of $\frac{1}{2 N \ln 2}$ from the objective function of (13) for the sake of convenience.

$I V-A$ Optimizing Alice's Transmit Power $\mathbf{P}_{\mathbf{a}}$

For Alice's transmit power optimization, the problem $(\mathrm{P})$ can be reformulated as

(P1) : $\max _{\mathbf{P}_{\mathbf{a}}} \sum_{n=1}^{N} \ln \left(1+\frac{a_{n} P_{a}[n]}{P_{a}[n]+b_{n}}\right)-\ln \left(1+\frac{c_{n} P_{a}[n]}{P_{a}[n]+d_{n}}\right)$

s.t. C5 and C6, where $a_{n}=\frac{\alpha[n]}{1-\alpha[n]}\left(1+P_{b}[n] h_{a b}[n]\right), b_{n}=\frac{P_{b}[n] h_{b a}[n]+1}{(1-\alpha[n]) h_{a b}[n]}, c_{n}=$ $\frac{\alpha[n]}{1-\alpha[n]}$, and $d_{n}=\frac{1}{(1-\alpha[n]) h_{a e}[n]}$. Using the following lemma, we see that the objective function of the problem $(\mathrm{P} 1)$ is in concave-minus-concave form.

Lemma 1. Define $f(x) \triangleq \ln \left(1+\frac{a x+b}{c x+d}\right), x \geq 0$ with positive constant values $a, b, c, d>0$ and subject to the condition ad $\geq$ bc. $f(x)$ is non-decreasing and has the first-order derivative given by

$$
f^{\prime}(x) \triangleq \frac{\partial f(x)}{\partial x}=\frac{a d-b c}{(c x+d)(x(a+c)+b+d)},
$$

By taking its second derivative, it can be obtained that the function $f(x)$ is concave as per the second-order condition law which states that $f(x)$ is concave iff $\frac{\partial^{2} f(x)}{\partial x^{2}} \leq 0$. Further, since the first order Taylor approximation at a given point $x_{0}$ of a concave function provides a global overestimator at that point [20], the inequality below holds

$$
\begin{aligned}
f(x) & \leq f\left(x_{0}\right)+f^{\prime}\left(x_{0}\right)\left(x-x_{0}\right) \\
& \leq \ln \left(1+\frac{a x_{0}+b}{c x_{0}+d}\right)+\frac{(a d-b c)\left(x-x_{0}\right)}{\left(c x_{0}+d\right)\left(b+d+(a+c) x_{0}\right)} .
\end{aligned}
$$

Therefore, we approximate the problem (P1) with the following convex alternative as

$$
\begin{aligned}
(\mathrm{P} 2): \max _{\mathbf{P}_{\mathbf{a}}} & \sum_{n=1}^{N} \ln \left(1+\frac{a_{n} P_{a}[n]}{P_{a}[n]+b_{n}}\right)-A_{n} P_{a}[n] \\
\text { s.t. } & \quad \text { C5 } \text { and } \mathrm{C} 6,
\end{aligned}
$$

where $A_{n}=\frac{c_{n} d_{n}}{\left(P_{a}^{(k)}[n]+d_{n}\right)\left(d_{n}+\left(c_{n}+1\right) P_{a}^{(k)}[n]\right)}$, with the feasible points $\mathbf{P}_{\mathbf{a}}^{(k)} \triangleq\left\{P_{a}^{(k)}[n]\right\}_{n=1}^{N}$. We note that the objective value of (P2) is lower-bounded by that of $(\mathrm{P} 1)$ at $\mathbf{P}_{\mathbf{a}}^{(k)}$. Since $(\mathrm{P} 2)$ is convex, thus, it can be solved optimally using CVX.

\section{IV-B Optimizing Bob's Transmit Power $\mathbf{P}_{\mathbf{b}}$}

By keeping the other variables unchanged, we optimize Bob's transmit power via reformulating the problem $(\mathrm{P})$, ignoring the constant second term of summation, as

$$
\begin{aligned}
(\mathrm{P} 3): \max _{\mathbf{P}_{\mathbf{b}}} & \sum_{n=1}^{N} \ln \left(1+\frac{k_{0} P_{b}[n]+k_{1}}{k_{2} P_{b}[n]+k_{3}}\right) \\
\text { s.t. } & \mathrm{C} 7 \text { and } \mathrm{C} 8,
\end{aligned}
$$

where $k_{0}=\alpha[n] P_{a}[n] h_{a b}^{2}[n], k_{1}=\alpha[n] P_{a}[n] h_{a b}[n], k_{2}=$ $h_{a b}[n]$, and $k_{3}=(1-\alpha[n]) P_{a}[n] h_{a b}[n]+1$. Following Lemma 1, (P3) is a convex problem, since it has concave objective function with convex constraints. Thus, it can be solved optimally via Lagrangian method as (P3) satisfies the Slater's condition and strong duality holds. As such, by temporarily dropping $\mathrm{C} 8$ and also letting $\tilde{\mathbf{P}}_{\mathbf{b}}$ and $\left(\tilde{\mathbf{P}}_{\mathbf{b}}, \lambda\right)$ be any primal and dual optimal points, only satisfying KarushKuhn-Tucker (KKT) conditions results in zero duality gap. As such, the Lagrangian of (P3) is given by

$\mathcal{L}\left(\mathbf{P}_{b}, \lambda\right)=-\sum_{n=1}^{N} \ln \left(1+\frac{k_{0} P_{b}[n]+k_{1}}{k_{2} P_{b}[n]+k_{3}}\right)+\lambda\left(\sum_{n=1}^{N} P_{b}[n]-P_{b}^{\text {tot }}\right)$,

where $\lambda \geq 0$ denotes the Lagrange multiplier associated with the inequality $\mathrm{C} 7$, and $P_{b}^{\text {tot }} \triangleq N \bar{P}_{b}$. Then, maximizing the 
Lagrangian dual function defined as $g(\lambda) \triangleq \inf _{\mathbf{P}_{\mathbf{b}}}\left\{\mathcal{L}\left(\mathbf{P}_{b}, \lambda\right)\right\}$, one can attain the optimality condition for $\forall n \in \mathbb{N}$ as

$$
\frac{k_{0} k_{3}-k_{1} k_{2}}{\left(k_{3}+k_{2} P_{b}[n]\right)\left(P_{b}[n]\left(k_{0}+k+2\right)\right)+k_{1}+k_{3}}-\lambda=0,
$$

Solving the above equation with respect to $P_{b}[n]$ and also taking into account constraint $\mathrm{C} 8$, leads to the closed-form analytical solution for (P3) as

$$
P_{b}^{\star}[n]=\min \left\{\hat{P}_{b}, \frac{\sqrt{a_{1}^{2}-4 a_{0} a_{2}}-a_{1}}{2 a_{2}}\right\}, \forall n \in \mathbb{N}
$$

where $a_{2}=k_{2}\left(k_{0}+k_{2}\right), a_{1}=k_{1} k_{2}+2 k_{2} k_{3}+k_{0} k_{3}$, and $a_{0}=$ $k_{3}\left(k_{1}+k_{3}\right)-\frac{k_{0} k_{3}-k_{1} k_{2}}{\lambda}$, wherein the non-negative Lagrange factor $\lambda$ can be obtained by applying a simple bisection search such that Bob's power budget constraint; i.e., $\sum_{n=1}^{N} P_{b}^{\star}[n] \leq$ $P_{b}^{t o t}$ is satisfied.

\section{IV-C Optimizing power allocation factor $\boldsymbol{\alpha}$}

To optimize the third block of variables $\alpha$, we recast the problem $(\mathrm{P})$ equivalently as

$$
\begin{aligned}
(\mathrm{P} 4): \max _{\boldsymbol{\alpha}} & \sum_{n=1}^{N} \ln (\Psi(\alpha[n])) \\
\text { s.t. } & \mathrm{C} 4: \quad 0 \leq \alpha[n] \leq 1, \quad \forall n \in \mathbb{N}
\end{aligned}
$$

where the function $\Psi(x)$ is defined as

$$
\Psi(x)=\frac{\left[(1-x) \gamma_{3}+1\right]\left[\left(x \gamma_{2}+1\right) \gamma_{1}+\gamma_{2}+1\right]}{\left(\gamma_{3}+1\right)\left[(1-x) \gamma_{1}+\gamma_{2}+1\right]},
$$

wherein $\gamma_{1} \triangleq P_{a}[n] h_{a b}[n], \gamma_{2} \triangleq P_{b}[n] h_{a b}[n], \gamma_{3} \triangleq$ $P_{a}[n] h_{a e}[n]$. We note that $\sum\left([\cdot]^{+}\right)$is a non-decreasing affine function and $\ln (\Psi(x))$ is a monotonically increasing function with respect to $\Psi(x)$. Therefore, $(\mathrm{P} 4)$ can be solved by maximizing every single term of the summation. Accordingly, optimized power allocation factor $\alpha^{\star}[n]$ for $\forall n \in \mathbb{N}$ can be calculated as

$$
\begin{aligned}
& \alpha^{\star}[n]=\frac{1+\gamma_{1}+\gamma_{2}}{\gamma_{1}}-\frac{\sqrt{\left(\gamma_{2} \gamma_{3}-\gamma_{1}\right)\left(1+\gamma_{2}\right)\left(1+\gamma_{1}+\gamma_{2}\right) \gamma_{2} \gamma_{3}}}{\gamma_{1} \gamma_{2} \gamma_{3}} \\
& \stackrel{(a)}{\approx} 1-\left[\sqrt{\frac{P_{b}[n]}{P_{a}[n]}\left(1+\frac{P_{b}[n]}{P_{a}[n]}\right)}-\frac{P_{b}[n]}{P_{a}[n]}\right]
\end{aligned}
$$

where $(a)$ follows from high SNR approximation, i.e., $\gamma_{i} \gg 1$ for $i \in\{1,2,3\}$.

Proof. We claim that $\Psi(x)$ is a quasi-concave function of $x$ in the feasible set $0 \leq x \leq 1$. Indeed, since the second derivative $\frac{\partial^{2} \Psi(x)}{\partial x^{2}}$ is quite sophisticated to argue about the convexity of the function, our approach is to use the properties of the first derivative of the function $\Psi(x)$ with respect to $x$. After tedious calculation, we can construe that for practical values of $\gamma_{1}, \gamma_{2}, \gamma_{3} \geq 1$, we have $\left.\frac{d \Psi}{d x}\right|_{0}=\frac{\gamma_{1}\left(\gamma_{2}+1\right)}{1+\gamma_{1}+\gamma_{2}}-\frac{\gamma_{3}}{\gamma_{3}+1}>0$, and $\left.\frac{d \Psi}{d x}\right|_{1}=\frac{\gamma_{1}+\frac{\gamma_{1}^{2}}{\gamma_{2}+1}-\gamma_{3}\left(1+\gamma_{1}\right)}{\gamma_{3}+1}<0$. Besides, it can be readily seen, via solving $\frac{\partial \Psi}{\partial x}=0$, that $\Psi(x)$ has only one extremum, say $x^{\star}$, in the domain $[0,1]$, leading to the fact that the function $\Psi(x)$ is increasing in $\left[0, x^{\star}\right)$, and tends to be a decreasing function in $\left(x^{\star}, 1\right]$, and thus, the proof is done.

\section{IV-D Optimizing UAV-Alice's trajectory $\omega_{a}$}

The corresponding subproblem to optimize Alice's trajectory is approximately reformulated, by taking the slack variables $\mathbf{s}=\{s[n]\}_{n=1}^{N}$ and $\mathbf{v}=\{v[n]\}_{n=1}^{N}$, as

$$
\begin{aligned}
&(\mathrm{P} 5): \max _{\boldsymbol{\omega}_{\boldsymbol{a}}, \mathbf{s}, \mathbf{v}} \sum_{n=1}^{N} \ln \left(c_{0}+\frac{c_{1}}{s[n]}\right)-\ln \left(1+\frac{c_{2}}{c_{3}+v[n]}\right) \\
& \text { s.t. } \mathrm{C} 1-\mathrm{C} 3, \\
& \mathrm{C} 9: \quad\left\|\omega_{a}[n]-\omega_{b}\right\|^{2} \leq s[n], \quad \forall n \in \mathbb{N} \\
& \text { C10: }\left\|\omega_{a}[n]-\omega_{e}\right\|^{2} \geq v[n], \quad \forall n \in \mathbb{N}
\end{aligned}
$$

where $c_{0}=\frac{\alpha[n] P_{a}[n]}{P_{b}[n]+P_{a}(1-\alpha[n])}, c_{1}=\frac{\alpha[n] P_{a}[n] P_{b}[n] \gamma_{0}}{P_{b}[n]+P_{a}(1-\alpha[n])}, c_{2}=$ $\alpha[n] P_{a}[n] \gamma_{0}, c_{3}=(1-\alpha[n]) P_{a}[n] \gamma_{0}$. Note that at the optimal point, constraints $\mathrm{C} 9$ and $\mathrm{C} 10$ must hold with equality, otherwise, by varying $s[n](v[n])$ the value of the objective function in (P5) increases, and this, of course, violates the optimality. Before solving (P5), we mention some fruitful lemmas below.

Lemma 2. Define the function $g(x) \triangleq \ln \left(a+b x^{-c}\right), x \geq 0$ with the first and second derivatives given respectively by

$$
\frac{\partial g(x)}{\partial x}=-\frac{b c}{a x^{c+1}+b x}, \frac{\partial^{2} g(x)}{\partial x^{2}}=\frac{b c\left(a(c+1) x^{c}+b\right)}{x^{2}\left(a x^{c}+b\right)^{2}},
$$

where the constants $a, b, c$ hold non-negative values. Having the non-negative second order derivative, the function $g(x)$ is convex, and since according to the first-order convexity condition which states that for a convex function the firstorder Taylor approximation is a global affine underestimator of that function and vice versa [20], therefore, $g(x)$ has the following global lower bound at the given point $x_{0}$ as

$$
g(x) \geq \ln \left(a+\frac{b}{x_{0}^{c}}\right)-\frac{b c}{x_{0}\left(a x_{0}^{c}+b\right)}\left(x-x_{0}\right),
$$

Lemma 3. Let $\mathbf{x}$ be a vector of variables $\left\{x_{i}\right\}_{i=1}^{N}$ and $\mathbf{a} \in \mathbb{R}^{N \times 1}$ be a constant vector. The function of negative norm-squared of this two vectors; $h(\mathbf{x})=\|\mathbf{x}-\mathbf{a}\|^{2}$, which obviously is a convex function with respect to the vector $\mathrm{x}$, has a global concave lower-bound, according to first-order convexity condition in Lemma 2, given by

$$
\|\mathbf{x}-\mathbf{a}\|^{2} \geq-\left\|\mathbf{x}_{\mathbf{0}}\right\|^{2}+2\left(\mathbf{x}_{\mathbf{0}}-\mathbf{a}\right)^{\dagger} \mathbf{x}+\|\mathbf{a}\|^{2},
$$

wherein $(\cdot)^{\dagger}$ represents the transpose operator.

Based on Lemma 2, we see that the objective function of problem (P5) has the convex-minus-convex form, and therefore, replacing the first convex term of which with the corresponding global concave approximation, similarly for the convex function $\left\|\omega_{a}[n]-\omega_{e}\right\|^{2}$ in constraint C10 using Lemma 3, yields an approximated lower-bound convex problem under the given feasible points $\omega_{\mathbf{a}}^{(k)} \triangleq\left\{\omega_{a}^{(k)}[n]\right\}_{n=1}^{N}$ $\mathbf{s}^{(k)} \triangleq\left\{s^{(k)}[n]\right\}_{n=1}^{N}$ as

$$
\begin{aligned}
&(\mathrm{P} 6): \max _{\boldsymbol{\omega}_{\boldsymbol{a}}, \mathbf{s} \mathbf{v}} \sum_{n=1}^{N} B_{n} s[n]-\ln \left(1+\frac{c_{2}}{c_{3}+v[n]}\right) \\
& \text { s.t. } \quad \mathrm{C} 1-\mathrm{C} 3, \mathrm{C} 9, \\
& \widetilde{C 10}: \quad v[n]-2\left(\omega_{a}^{(k)}[n]-\omega_{e}\right)^{\dagger} \omega_{a}[n]+c_{4} \leq 0,
\end{aligned}
$$




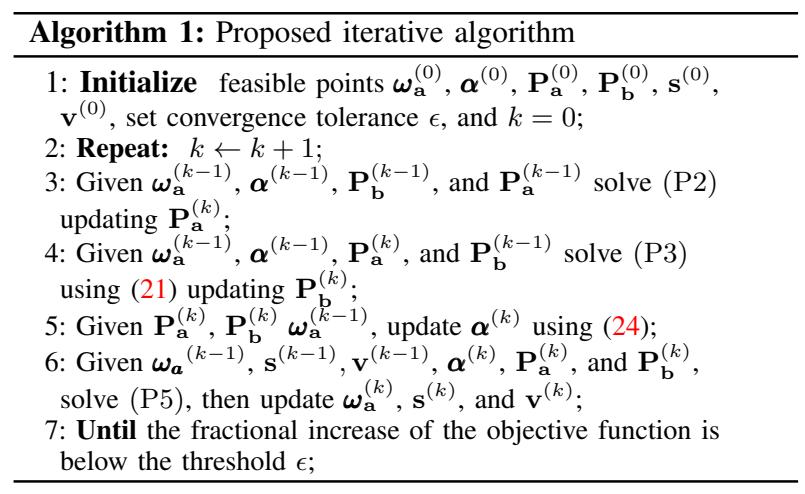

where $B_{n}=-\frac{c_{1}}{s^{(k)}[n]\left(c_{0}+c_{1}\right)}, c_{4} \triangleq\left\|\omega_{a}^{(k)}[n]\right\|^{2}-\left\|\omega_{e}\right\|^{2}$. Being a convex problem, (P6) can be solved efficiently using CVX.

\section{IV-E Overall Algorithm}

The proposed iterative algorithm is summarized in Algorithm 1 , whose complexity is dominated by the complexity of each sub-problem. As such, sub-problem (P1) is solved by SCA method and its complexity mainly relies on the number of variables and constraints. Since there are $N+1$ constraints in $(\mathrm{P} 2)$, the number of iterations required for SCA is $\mathcal{O}\left(\sqrt{N+1} \log _{2}\left(\frac{1}{\varepsilon_{1}}\right)\right)$, where $\varepsilon_{1}$ is the accuracy of SCA method for solving (P2). Besides, the complexity of solving (P2) at each iteration is $\mathcal{O}\left(N^{2}(N+1)\right)$. Consequently, the complexity of solving (P2) can be approximately represented as $\mathcal{O}\left(N^{3.5} \log _{2}\left(\frac{1}{\varepsilon_{1}}\right)\right)$. We solved (P3) semi-analytically based on Lagrangian method and using a bisection search whose complexity is $\log _{2}\left(\frac{\varepsilon_{0}}{\varepsilon_{3}}\right)$, where $\varepsilon_{0}$ and $\varepsilon_{3}$ represent initial bracket size of $\left[\lambda_{\min }, \lambda_{\max }\right]$, and the required tolerance, respectively. Further, (P4) is solved optimally at one iteration. Similar to that of (P1), the complexity of (P5) is approximately $\mathcal{O}\left((4 N)^{2}(3 N+1)^{1.5} \log _{2}\left(\frac{1}{\varepsilon_{2}}\right)\right)$. Finally, the overall computational complexity of the proposed algorithm is approximately $\mathcal{O}\left(M\left(N^{3.5} \log _{2}\left(\frac{1}{\varepsilon_{1}}\right)+\log _{2}\left(\frac{\varepsilon_{0}}{\varepsilon_{3}}\right)+\right.\right.$ $\left.\left.48 \sqrt{3} N^{3.5} \log _{2}\left(\frac{1}{\varepsilon_{2}}\right)\right)\right)$, wherein $M$ denotes the number of iterations for Algorithm 1, which is inversely proportional to the termination threshold $\epsilon$.

For convergence analysis of Algorithm 1, we define the objective values of original problem $(\mathrm{P})$, the subproblems problems (P2), and (P6) at iteration $k$ as $\bar{R}_{\text {sec }}\left(\mathbf{P}_{\mathbf{a}}^{k}, \mathbf{P}_{\mathbf{b}}^{k}, \boldsymbol{\alpha}^{k}, \boldsymbol{\omega}_{\mathbf{a}}^{k}\right)$, $\Theta_{l b}\left(\mathbf{P}_{\mathbf{a}}^{k}, \mathbf{P}_{\mathbf{b}}^{k}, \boldsymbol{\alpha}^{k}, \boldsymbol{\omega}_{\mathbf{a}}^{k}\right)$ and $\Xi_{l b}\left(\mathbf{P}_{\mathbf{a}}^{k}, \mathbf{P}_{\mathbf{b}}^{k}, \boldsymbol{\alpha}^{k}, \boldsymbol{\omega}_{\mathbf{a}}^{k}\right)$, respectively. We can write as

$$
\begin{aligned}
& \bar{R}_{\text {sec }}\left(\mathbf{P}_{\mathbf{a}}^{k}, \mathbf{P}_{\mathbf{b}}^{k}, \boldsymbol{\alpha}^{k}, \boldsymbol{\omega}_{\mathbf{a}}^{k}\right) \stackrel{\left(a_{1}\right)}{\leq} \bar{R}_{s e c}\left(\mathbf{P}_{\mathbf{a}}^{k+1}, \mathbf{P}_{\mathbf{b}}^{k}, \boldsymbol{\alpha}^{k}, \boldsymbol{\omega}_{\mathbf{a}}^{k}\right) \\
& \stackrel{\left(a_{2}\right)}{\leq} \bar{R}_{s e c}\left(\mathbf{P}_{\mathbf{a}}^{k+1}, \mathbf{P}_{\mathbf{b}}^{k+1}, \boldsymbol{\alpha}^{k}, \boldsymbol{\omega}_{\mathbf{a}}^{k}\right) \stackrel{(b)}{=} \Theta_{l b}\left(\mathbf{P}_{\mathbf{a}}^{k+1}, \mathbf{P}_{\mathbf{b}}^{k+1}, \boldsymbol{\alpha}^{k}, \boldsymbol{\omega}_{\mathbf{a}}^{k}\right) \\
& \stackrel{(c)}{\leq} \bar{R}_{\text {sec }}\left(\mathbf{P}_{\mathbf{a}}^{k+1}, \mathbf{P}_{\mathbf{b}}^{k+1}, \boldsymbol{\alpha}^{k+1}, \boldsymbol{\omega}_{\mathbf{a}}^{k}\right) \stackrel{(d)}{=} \Xi_{l b}\left(\mathbf{P}_{\mathbf{a}}^{k+1}, \mathbf{P}_{\mathbf{b}}^{k+1}, \boldsymbol{\alpha}^{k+1}, \boldsymbol{\omega}_{\mathbf{a}}^{k}\right) \\
& \stackrel{(e)}{\leq} \bar{R}_{\text {sec }}\left(\mathbf{P}_{\mathbf{a}}^{k+1}, \mathbf{P}_{\mathbf{b}}^{k+1}, \boldsymbol{\alpha}^{k+1}, \boldsymbol{\omega}_{\mathbf{a}}^{k+1}\right),
\end{aligned}
$$

where $\left(a_{1}\right)$ and $\left(a_{2}\right)$ follows from the definition of the optimal solution to (P1) and (P3), respectively. Besides, the equalities (b) and $(d)$ hold since the first order Taylor approximation is adopted and that the objective functions of (P2) and (P6) share the same value with the original function at the given points, respectively. Finally, $(c)$ and $(e)$ hold since the objective functions of (P2) and (P6) are tight lower-bounds to that of
TABLE I: Simulation parameters

\begin{tabular}{||c|c||c|c||c|c||}
\hline Notation & Value & Notation & Value & Notation & Value \\
\hline \hline$H$ & $100 \mathrm{~m}$ & $\gamma_{0}$ & $80 \mathrm{~dB}$ & $\lambda$ & 0.5 \\
\hline$\omega_{b}$ & $(0,0,0)$ & $P_{a v e}$ & $0 \mathrm{dBm}$ & $\bar{\alpha}$ & 0.5 \\
\hline$\omega_{e}$ & $(100,0,0)$ & $\hat{P}_{a}=\hat{P}_{b}$ & $4 \bar{P}_{\text {ave }}$ & $\epsilon$ & $10^{-4}$ \\
\hline$\omega_{a i}$ & $(50,+200,0)$ & $P_{a}$ & $\lambda P_{\text {ave }}$ & $V$ & $4 \mathrm{~m} / \mathrm{s}$ \\
\hline$\omega_{a f}$ & $(50,-200,0)$ & $\bar{P}_{b}$ & $(1-\lambda) P_{a v e}$ & $N$ & 100 \\
\hline \hline
\end{tabular}

(P1) and (P5), respectively. The last inequality in (30) indicates that the objective value of $(\mathrm{P})$ is non-decreasing with the iteration index. Since the optimal value of $(\mathrm{P})$ is upper bounded by a finite value, Algorithm 1 is guaranteed to converge.

\section{NUMERICAL RESULTS}

In this section, we conduct simulations to verify the convergence of Algorithm 1 and demonstrate the performance of the proposed scheme, labelled as JTDORA. Unless otherwise stated, we adopt all parameters in Table I for simulations. Besides, for initial feasible trajectory of UAV, we adopt the best-effort approach (labeled as Baseline trajectory), wherein given Alice's flying duration $T$ seconds, she flies towards Bob with maximum speed in direct path, then hovers right above Bob's location as long as possible, and finally heads directly at maximum speed to the final location. Otherwise, when the hovering phase is impossible due to limited time, while flying at maximum speed to Bob, Alice turns at a midway point to reach the final location by the end of the time. We consider the following benchmarks for performance comparison:

- ANOPC: AN-based scheme with baseline trajectory, fixed AN injection factor, i.e., $\alpha[n]=\bar{\alpha}, \forall n$, and iteratively optimizing $\mathbf{P}_{\mathbf{a}}$ and $\mathbf{P}_{\mathbf{b}}$ using (P2) and (21), respectively. Note that ignoring trajectory design, this scheme is similar to that in [17].

- ANTD: AN-based scheme with trajectory design via iteratively optimizing $\omega_{\mathrm{a}}$ using (P6), fixed transmit powers, $P_{a}[n]=\bar{P}_{a}, \forall n$, and $P_{b}[n]=\bar{P}_{b}, \forall n$, and equal AN injection factor $\alpha[n]=\bar{\alpha}, \forall n$.

- ANERA: AN-based scheme with baseline trajectory and fixed resource allocations, i.e., $P_{a}[n]=\bar{P}_{a}, P_{b}[n]=$ $\bar{P}_{b}, \alpha[n]=\bar{\alpha}, \forall n$. This scheme is indeed the initial feasible point $\left(\boldsymbol{\omega}_{\mathbf{a}}^{(0)}, \mathbf{P}_{\mathbf{a}}^{(0)}, \mathbf{P}_{\mathbf{b}}^{(0)}, \boldsymbol{\alpha}^{(0)}\right)$ used for solving Algorithm 1.

- TDPC: Trajectory optimization and power controlling without $\mathrm{AN}$ allocation, i.e., $\alpha[n]=0 \forall n$. It is quite similar, excluding the duplexing aspect, to that in [5]. For the fairness of comparisons, we set $\bar{P}_{a}=P_{\text {ave }}$ to ensure all schemes have an equal total transmission power budget.

We verify our analysis in Fig. 2a by plotting ASR vs iteration indices. We see that the ASR is non-decreasing over the iteration index, and our proposed algorithm converges in very few iterations. Besides, the more the mission time, the higher the achievable ASR, since Alice can spend longer time hovering near Bob. In Fig. $2 \mathrm{~b}$ we plot the designed trajectory using Algorithm 1 vs the baseline trajectory for different flight durations. When $T=100 \mathrm{~s}$, the only feasible path is the direct line from initial to final locations and Alice does not have flexibility in path planning. When $T$ increases, the designed trajectory of Alice gets curved, since this makes Alice fly as close as possible to Bob and as farther as possible from Eve to improve ASR. Note that for baseline trajectory when approximately $T \leq 104 \mathrm{~s}$ holds, Alice can just perform the non-stop midpoint-turn flying approach; otherwise, she can do 


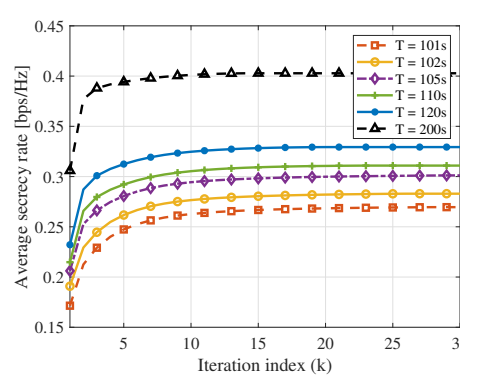

(a) Convergence Verification.

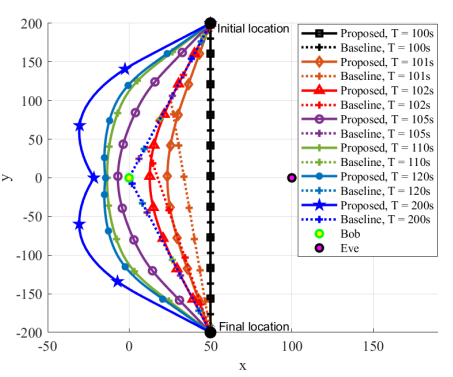

(b) Alice's designed trajectory.

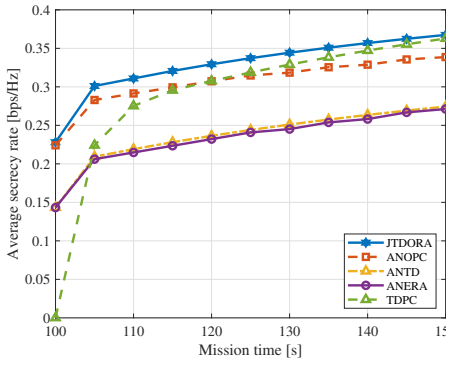

(c) ASR vs. mission time.

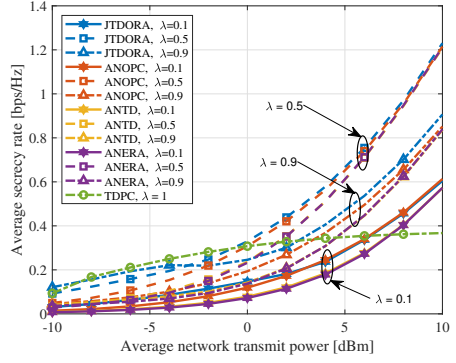

(d) ASR vs. Pave.

Fig. 2: ASR performance of proposed efficient solution and designed UAV's trajectory according to Algorithm 1.

fly-hover-fly with different hovering durations. From Fig. 2b, we observe, using the JTDORA-based trajectory design, the best location for Alice to have a secure communication while hovering, is quite farther from Bob, depending on the mission duration. In Fig. 2c, we compare the performance of the proposed JTDORA with the aforementioned ANOPC, ANTD, ANERA, and TDPC benchmark schemes in terms of ASR for different flight durations. For AN-based schemes, we set equal total power allocation, denoted by $\lambda$, between Alice and Bob for information and AN transmission, respectively. We observe that, for the mission times of interest, our proposed JTDORA demonstrates the highest ASR amongst all. In particular, when $T \leq 104 \mathrm{~s}, T D P C$ without AN performs the worst, but when $T$ is high (e.g. $T>104 \mathrm{~s}$ ), $T D P C$ achieves improved ASR, when compared to ANOPC, ANTD, and ANERA, since TDPC consumes all transmit power for information transmission. For a large $T$, when UAV is able to find the most convenient location for communication, TDPC can highly improve ASR. In contrast, the AN-based schemes spend some fraction of allocated power for AN injection, leaving less power for confidential information transmission. Overall, we conclude that our proposed JTDORA scheme is more appropriate for stringent flight mission periods, and $\lambda$ should be chosen dynamically according to the flight duration. Fig. $2 \mathrm{~d}$ depicts the impacts of average network power $P_{\text {ave }}$ on ASR for different schemes with different power ratio factors, with $T=120 \mathrm{~s}$. We observe that increasing $P_{\text {ave }}$ results in improved ASR for all schemes, whereas for TDPC, an ASR ceiling appears in very high average network transmit power that limits its ASR performance. Further, we see that at high $P_{\text {ave }}$, e.g., $P_{\text {ave }}=10$ $\mathrm{dBm}$, Alice's maneuverability has less impact on the ASR for $\lambda=0.5$. Also, the ASR performance for AN-based schemes is sensitive to power ratio $\lambda$, and higher power ratio $\lambda=0.9$ for lower $P_{\text {ave }}$ values, yields best ASR. This implies that when the total transmit power is low, the higher fraction should be dedicated to Alice's transmission, while for higher amounts of $P_{t o t}$, equal power allocation $(\lambda=0.5)$ is appealing, to achieve the best ASR for JTDORA

In this paper, we presented a secure two-phase transmission protocol via AN-assisted UAV communications. To combat eavesdropping, we formulated an ASR optimization problem in terms of path planning and resource allocation, and then proposed an iterative algorithm to solve it. The proposed computationally efficient algorithm provides suboptimal solution in terms of network transmit power, UAV's flying trajectory, and AN injection factor in order to maximize the ASR. We evaluated our proposed scheme via simulations in terms of ASR and flying trajectory, and demonstrated its effectiveness. REFERENCES

[1] X. Sun, D. W. K. Ng, Z. Ding, Y. Xu, and Z. Zhong, "Physical layer security in uav systems: Challenges and opportunities," IEEE Wireless Commun., vol. 26, no. 5, pp. 40-47, 2019.

[2] J. M. Hamamreh, H. M. Furqan, and H. Arslan, "Classifications and applications of physical layer security techniques for confidentiality: A comprehensive survey," IEEE Commun. Surveys Tuts., vol. 21, no. 2 , pp. $1773-1828,2018$

[3] M. Tatar Mamaghani, A. Kuhestani, and K.-K. Wong, "Secure two-way transmission via wireless-powered untrusted relay and external jammer," IEEE Trans. Veh. Technol., vol. 67, no. 9, pp. 8451-8465, 2018.

[4] Q. Wu, W. Mei, and R. Zhang, "Safeguarding wireless network with uavs: A physical layer security perspective," IEEE Wireless Commun., vol. 26, no. 5, pp. 12-18, 2019.

[5] G. Zhang, Q. Wu, M. Cui, and R. Zhang, "Securing uav communications via joint trajectory and power control," IEEE Trans. Wireless Commun., vol. 18, no. 2, pp. 1376-1389, 2019.

[6] M. Cui, G. Zhang, Q. Wu, and D. W. K. Ng, "Robust trajectory and transmit power design for secure uav communications," IEEE Trans. Veh. Technol., vol. 67, no. 9, pp. 9042-9046, 2018.

[7] Q. Wang, Z. Chen, W. Mei, and J. Fang, "Improving physical layer security using uav-enabled mobile relaying," IEEE Wireless Commun. Lett., vol. 6, no. 3, pp. 310-313, 2017.

[8] Q. Wang, Z. Chen, H. Li, and S. Li, "Joint power and trajectory design for physical-layer secrecy in the uav-aided mobile relaying system," IEEE Access, vol. 6, pp. 62 849-62 855, 2018.

[9] L. Xiao, Y. Xu, D. Yang, and Y. Zeng, "Secrecy energy efficiency maximization for uav-enabled mobile relaying," IEEE Trans. Green Commun. Netw., 2019.

[10] M. Tatar Mamaghani and Y. Hong, "On the performance of low-altitude uav-enabled secure af relaying with cooperative jamming and swipt," IEEE Access, vol. 7, pp. 153060-153073, 2019.

[11] A. Li, Q. Wu, and R. Zhang, "Uav-enabled cooperative jamming for improving secrecy of ground wiretap channel," IEEE Wireless Commun. Lett., vol. 8, no. 1, pp. 181-184, 2018.

[12] Y. Cai, Z. Wei, R. Li, D. W. K. Ng, and J. Yuan, "Joint trajectory and resource allocation design for energy-efficient secure uav communication systems," IEEE Trans. Commun., vol. 68, no. 7, pp. 4536-4553, 2020.

[13] M. Tatar Mamaghani and Y. Hong, "Improving phy-security of uavenabled transmission with wireless energy harvesting: Robust trajectory design and communications resource allocation," IEEE Trans. Veh. Technol., vol. 69, no. 8, pp. 8586-8600, 2020.

[14] Y. Li, R. Zhang, J. Zhang, S. Gao, and L. Yang, "Cooperative jamming for secure uav communications with partial eavesdropper information," IEEE Access, vol. 7, pp. 94 593-94603, 2019.

[15] S. Goel and R. Negi, "Guaranteeing secrecy using artificial noise," IEEE Trans. Wireless Commun., vol. 7, no. 6, p. 2180-2189, June 2008.

[16] S. Liu, Y. Hong, and E. Viterbo, "Artificial noise revisited," IEEE Trans. Inf. Theory, vol. 61, no. 7, pp. 3901-3911, 2015.

[17] B. He, Y. She, and V. K. N. Lau, "Artificial noise injection for securing single-antenna systems," IEEE Trans. Veh. Technol., vol. 66, no. 10, pp. 9577-9581, 2017.

[18] G. Zhang, Q. Wu, M. Cui, and R. Zhang, "Securing uav communications via joint trajectory and power control," IEEE Trans. Wireless Commun., vol. 18, no. 2, pp. 1376-1389, 2019.

[19] X. Lin et al., "The sky is not the limit: Lte for unmanned aerial vehicles," IEEE Commun. Magazine, vol. 56, no. 4, pp. 204-210, 2018.

[20] S. Boyd and L. Vandenberghe, Convex Optimization. Cambridge university press, 2004. 